\title{
Periodic expansion in determining minimal sets of Lefschetz periods for Morse-Smale diffeomorphisms
}

\author{
Grzegorz Graff(i), Małgorzata Lebiedź(i) and Adrian Myszkowski(i)
}

\begin{abstract}
We apply the representation of Lefschetz numbers of iterates in the form of periodic expansion to determine the minimal sets of Lefschetz periods of Morse-Smale diffeomorphisms. Applying this approach we present an algorithmic method of finding the family of minimal sets of Lefschetz periods for $N_{g}$, a non-orientable compact surfaces without boundary of genus $g$. We also partially confirm the conjecture of Llibre and Sirvent (J Diff Equ Appl 19(3):402-417, 2013) proving that there are no algebraic obstacles in realizing any set of odd natural numbers as the minimal set of Lefschetz periods on $N_{g}$ for any $g$.
\end{abstract}

Mathematics Subject Classification. Primary 37D15, 37C25, 37E15.

Keywords. Morse-Smale diffeomorphism, Lefschetz number, periodic expansion, zeta function, set of periods, non-orientable compact surfaces.

\section{Introduction}

Let $f: M \rightarrow M$ be a Morse-Smale diffeomorphism, where $M$ is a compact manifold without boundary. Morse-Smale diffeomorphisms, structurally stable and having relatively simple dynamics, constitute an important subclass of diffeomorphisms that were carefully studied during past decades (cf. [17] and the references therein).

One of the problems studied for Morse-Smale diffeomorphisms is the structure of the set of its minimal periods. The promising results in this direction may be obtained by the comparison (via Lefschetz-Hopf theorem) of the global behavior of $f$ expressed by Lefschetz numbers of iterates $\left(L\left(f^{n}\right)_{n}\right)$ and local properties of $f$ near periodic points represented by local fixed point indices of iterates. Basing on this relation $\operatorname{MPer}_{L}(f)$ called minimal set of Lefschetz periods is considered (cf. Definition 3.3). $\operatorname{MPer}_{L}(f)$ provides the information about the set of periodic points of $f$ as it is the subset of minimal

Research supported by the National Science Centre, Poland, UMO-2014/15/B/ST1/01710. 
periods of $f$. The description of $\operatorname{MPer}_{L}(f)$ was performed (in dependence on the behavior of $f_{*}$, a map induced by $f$ on homology groups) for the sphere $S^{n}$ [21], orientable compact surfaces [29], non-orientable compact surfaces [28], disk with $n$ holes [19], product of $l$-dimensional spheres [3], $n$-dimensional torus $T^{n}$ [23]. Let us mention here that using similar approach the set of periods as well as so-called minimal set of periods of the Morse-Smale diffeomorphisms for $T^{2}$ was characterized in [22].

The method of determining $\operatorname{MPer}_{L}(f)$ in the above papers is based on the decomposition of Lefschetz zeta function into all possible factors and taking the exponents which are present in all such decompositions. However, this is sometimes not an easy task, especially in case when the dimensions of homologies are large. The purpose of this paper was to present another approach, which simplifies calculations a lot. Namely, we use the language of so-called periodic expansions, which enables us to represent the sequences of Lefschetz numbers and fixed point indices of iterates in the convenient form of the combination of some basic $k$-periodic sequences $\left(\operatorname{reg}_{k}\right)_{n}[1,25,30]$. Using this apparatus we are able to reformulate the definition of $\operatorname{MPer}_{L}(f)$ and to express it by decompositions of Lefschetz numbers into sums of $\left(\operatorname{reg}_{k}\right)_{n}$, each of which represents, for odd $k$, a periodic orbit of minimal period $k$. As a consequence, we prove that $\operatorname{MPer}_{L}(f)$ is equal to the set of all non-zero odd $k$ that appear in such decomposition (Theorem 6.2).

Our method is equivalent to the approach based on Lefschetz zeta functions but is computationally much simpler. We also provide the general form of sequences of Lefschetz numbers of iterates for Morse-Smale diffeomorphisms (Sect. 4.1), which could be a useful device for other investigations. In Sect. 7 we show the advantages of our approach considering self-maps of $N_{g}$, a non-orientable compact surface without boundary of genus $g$, and confirming the algebraic part of the problem of the realization of elements of $\operatorname{MPer}_{L}(f)$ for self-maps of $N_{g}$ (Conjecture 7.1) from [28].

In the final part of the paper we also provide an algorithm for computing the minimal sets of Lefschetz periods for self-maps of $N_{g}$. We verify by a computer program $\operatorname{MPer}_{L}(f)$ for $g<10$ found in [28] correcting one omission and compute $\operatorname{MPer}_{L}(f)$ for higher values of $g$.

Finally, let us mention that all the obtained results remain valid not only for Morse-Smale diffeomorphisms, but also for a larger class of maps, namely for maps with finitely many periodic points all of them hyperbolic (cf. Remark 6.3).

\section{The class of Morse-Smale diffeomorphisms}

In this section we recall some basic definitions related to the class of MorseSmale diffeomorphisms.

Let $f: X \rightarrow X$, by $\mathrm{P}(f)$ we will denote the set of periodic points of $f$. Let us remind that $x$ is a periodic point with minimal period $n$ if $f^{n}(x)=x$ and $f^{m}(x) \neq x$ for $m<n$ (we will also call $x$ an $n$-periodic point for short). 
The set of all $n$-periodic points will be denoted by $\mathrm{P}_{n}(f)$ and the set of all minimal periods by $\operatorname{MPer}(f)$.

Definition 2.1. Let $f: M \rightarrow M$ be a $C^{1}$ diffeomorphism of a manifold $M$. A point $x \in M$ will be called non-wandering if for any neighbourhood $U$ of $x$ there exists an integer $n>1$ such that $f^{n}(U) \cap U \neq \emptyset$. The set of all non-wandering points of $f$ will be denoted by $\Omega(f)$.

Definition 2.2. Let $f: M \rightarrow M$ be a $C^{1}$ diffeomorphism of a manifold $M$ and $x$ be an $n$-periodic point. Then $x$ is called hyperbolic if none of the eigenvalues of the derivative $D f(x)$ of $f$ at $x$ has modulus equal to 1 .

Definition 2.3. For a hyperbolic $n$-periodic point $x$ we define a stable manifold $W^{s}(x)$ as

$$
W^{s}(x)=\left\{y \in X: f^{n k}(y) \rightarrow x \text { as } k \rightarrow \infty\right\}
$$

and an unstable manifold $W^{u}(x)$ as

$$
W^{u}(x)=\left\{y \in X: f^{n k}(y) \rightarrow x \text { as } k \rightarrow-\infty\right\} .
$$

Definition 2.4. Let $M$ be a compact manifold. A diffeomorphism $f: M \rightarrow M$ is Morse-Smale if

(i) $\Omega(f)$ is finite,

(ii) all periodic points are hyperbolic,

(iii) for each $x, y \in \mathrm{P}, W^{s}(x)$ and $W^{u}(y)$ have transversal intersections.

Remark 2.5. It follows from $(i)$ that the set $\Omega(f)$ consists only of periodic points, i.e. $\Omega(f)=\mathrm{P}(f)$.

\section{Lefschetz numbers of iterates and Lefschetz zeta function}

First we remind the definition of Lefschetz numbers of iterates; for simplicity we will consider homology with rational coefficients.

Let $K$ be a CW-complex of dimension $m$ with the homology groups $H_{i}(K ; \mathbb{Q})$, where $i=0,1, \ldots, m$. In case the homology coefficients are equal to $\mathbb{Q}$ the groups $H_{i}(K ; \mathbb{Q})$ are finite dimensional linear spaces over $\mathbb{Q}$. For a self-map $f$ of $K$ we denote by $f_{* i}$ the linear map induced by $f$ on $H_{i}(K ; \mathbb{Q})$ and by $f_{*}$ the self-map $\bigoplus_{i=0}^{m} f_{* i}$ of $\bigoplus_{i=0}^{m} H_{i}(K ; \mathbb{Q})$. The Lefschetz number $L\left(f^{n}\right)$ of $f^{n}$ is then equal to

$$
L\left(f^{n}\right)=\sum_{i=0}^{m}(-1)^{i} \operatorname{tr}\left(f^{n}\right)_{* i},
$$

where $\operatorname{tr}\left(f^{n}\right)_{* i}$ is the trace of the integer matrix representing $\left(f^{n}\right)_{* i}: H_{i}(K ; \mathbb{Q})$ $\rightarrow H_{i}(K ; \mathbb{Q})$. Notice that if $A$ is a matrix of $f_{* i}$, then $A^{n}$ is a matrix of $\left(f^{n}\right)_{* i}$, representing the homomorphism induced on $H_{i}(K ; \mathbb{Q})$ by $f^{n}$ (cf. [25]).

Lefschetz zeta function, $Z_{f}$, which is a useful tool in periodic points theory, codes information on the whole sequence of Lefschetz numbers of iterates:

$$
Z_{f}(t)=\exp \left(\sum_{n=1}^{\infty} \frac{L\left(f^{n}\right)}{n} t^{n}\right) .
$$


We will also consider zeta function associated with a given integer sequence $\left(a_{n}\right)_{n}$, which will be defined in an analogous way:

$$
Z_{a_{n}}(t)=\exp \left(\sum_{n=1}^{\infty} \frac{a_{n}}{n} t^{n}\right) .
$$

Remark 3.1. An alternative formula for the Lefschetz zeta function may be given by a use of eigenvalues of $f_{*}$, namely if $\lambda_{i}$ is an eigenvalue of $f_{*}$, taken with algebraic multiplicities $k_{i}$, then

$$
Z_{f}(t)=\prod_{i}\left(1-\lambda_{i} z\right)^{(-1)^{m_{i}+1} k_{i}},
$$

where $m_{i}$ denotes the index of the homology group associated with $\lambda_{i}[25]$.

It turns out that the Lefschetz zeta function for Morse-Smale diffeomorphisms has very special form, first described by Franks in [9].

Let $M$ be a smooth manifold and $x$ be a hyperbolic $p$-periodic point of a map $f: M \rightarrow M$. Denote by $E_{x}^{u n}$ the subspace of the tangent $T M_{x}$ spanned by eigenvectors of $D f^{p}(x)$ which correspond to eigenvalues which are greater than one in absolute value. Let $\gamma$ be an orbit of $x$, we define $u=\operatorname{dim} E_{x}^{u n}$ and $\Delta$, the orientation type of $\gamma$, as +1 if $D f^{p}(x)$ preserves the orientation and -1 if it reverses the orientation.

By $\Sigma$ we denote periodic data, i.e. a collection of all the triples $(p, u, \Delta)$ which corresponds to periodic orbits of $f$.

Theorem 3.2. [9] Let $M$ be a closed manifold, and $f: M \rightarrow M$ be a $C^{1}$ map with finitely many periodic points, all of them hyperbolic; then

$$
Z_{f}(t)=\prod_{(p, u, \Delta) \in \Sigma}\left(1-\Delta t^{p}\right)^{(-1)^{u+1}},
$$

where $(p, u, \Delta)$ belongs to periodic data of $f$.

Definition 3.3. Let $Z_{f}(t) \neq 1$, the minimal set of Lefschetz periods of $f$ is defined as

$$
\operatorname{MPer}_{L}(f):=\bigcap\left\{r_{1}, \ldots, r_{N(f)}\right\},
$$

where the intersection is considered over all the possible expressions of $Z_{f}(t)$ in the following form:

$$
Z_{f}(t)=\prod_{i=1}^{N(f)}\left(1+\Delta_{i} t^{r_{i}}\right)^{m_{i}},
$$

where $\Delta_{i} \in\{-1,1\}, r_{i}$ are positive integer values, $m_{i}$ are nonzero integers and $N(f)$ is a positive integer that depends on the function $f$. For $Z_{f}(t)=1$ we define $\operatorname{MPer}_{L}(f):=\emptyset$.

The importance of the minimal sets of Lefschetz periods for MorseSmale diffeomorphisms results from the following fact, which is a straightforward consequence of Theorem 3.2

$$
\operatorname{MPer}_{L}(f) \subset \operatorname{MPer}(f) .
$$

Now, we introduce the notion of quasi-unipotent maps. 
Definition 3.4. A rational linear transformation is called quasi-unipotent if their eigenvalues are roots of unity. We will call a continuous map $f: M \rightarrow M$ quasi-unipotent if the maps $f_{*_{k}}$ are quasi-unipotent for $0 \leq k \leq m$, where $m$ is the dimension of the manifold $M$.

The following fact makes it possible to determine Lefschetz zeta function for a Morse-Smale diffeomorphism in a relatively easy way.

Proposition 3.5 [32]. Let $f$ be a Morse-Smale diffeomorphism of a compact manifold, then $f$ is quasi-unipotent.

Due to Proposition 3.5 and the formula (3.4) Lefschetz zeta function for a Morse-Smale diffeomorphism may be expressed as a rational function with the nominator and denominator being a product of cyclotomic polynomials, whose degrees are bounded by the dimensions of homology spaces. As for a given $n$ there is a finite number of cyclotomic polynomials of degree $\leq n$, for a given manifold $M$ there is a finite number of different forms of zeta functions on $M$. This observation was a base for a strategy of finding minimal set of Lefschetz periods for all Morse-Smale diffeomorphisms on a given manifold $M$, which may be described in the following steps. For a given Morse-Smale diffeomorphism $f$ find zeta functions $Z_{f}(t)$ (expressed in terms of cyclotomic polynomials), next determine all their decompositions into the products of elements of the form (3.6), and finally take the common part of coefficients $r_{i}$ (which are related to minimal periods of $f$ ) over all such products. We proposed below the alternative strategy which is based on representing $\left(L\left(f^{n}\right)\right)_{n}$ as the sum of basic periodic sequences and decomposing it into sum of sequences related to periodic orbits of the considered map.

\section{Periodic expansion of Lefschetz numbers of iterates}

Definition 4.1. A sequence of integer numbers $\left(a_{n}\right)_{n=1}^{\infty}$ will be called a Dold sequence if the following congruences (called Dold congruences or Dold relations) are fulfilled:

$$
\sum_{k \mid n} \mu(k) a_{\frac{n}{k}} \equiv 0(\bmod n) \quad \text { for each } n \geq 1,
$$

where $\mu: \mathbb{N} \rightarrow \mathbb{Z}$ is the classical Möbius function, given by the following formula:

$$
\mu(n)= \begin{cases}1 & \text { if } n=1 \\ (-1)^{k} & \text { if } n=p_{1} p_{2} \cdots p_{k} \text { for different primes } p_{i} \\ 0 & \text { otherwise. }\end{cases}
$$

Dold sequences play important role not only in dynamics but also in number theory (cf. $[2,16]$ ). There is a convenient way of writing down a Dold sequence by using so-called periodic expansion, i.e. representing the sequence as a combination of some basic periodic sequences. 
Definition 4.2. Let $k$ be a fixed natural number. We define

$$
\operatorname{reg}_{k}(n)= \begin{cases}k & \text { if } k \mid n, \\ 0 & \text { if } k \nmid n\end{cases}
$$

Thus, $\operatorname{reg}_{k}$ is the periodic sequence:

$$
(0, \ldots, 0, k, 0, \ldots, 0, k, \ldots)
$$

where the non-zero entries appear for indices divisible by $k$.

Proposition 4.3 (Proposition 2.7 in [30]). Any arithmetic function $\left(a_{n}\right)_{n=1}^{\infty}$ can be written uniquely in the following form of a periodic expansion:

$$
a_{n}=\sum_{k=1}^{\infty} b_{k} \operatorname{reg}_{k}(n), \text { where } b_{n}=\frac{1}{n} \sum_{k \mid n} \mu\left(\frac{n}{k}\right) a_{k} \in \mathbb{Q} .
$$

Moreover, $\left(a_{n}\right)_{n}$ is integral valued and satisfies Dold congruences iff $b_{k} \in \mathbb{Z}$ for every $k \in \mathbb{N}$.

Remark 4.4. By Proposition 4.3 every Dold sequence is an integral combination (possibly infinite) of basic sequences $\operatorname{reg}_{k}$ (with coefficients $b_{k}$ which are called Dold coefficients).

There is a deep relation between sequences $\left(a_{n}\right)_{n}$ and $\left(b_{n}\right)_{n}$ that appear in Proposition 4.3, which may be expressed in the language of formal power series and formal products.

Theorem 4.5 (Theorem 2 in [6]). Let $\left(a_{n}\right)_{n},\left(b_{n}\right)_{n}$ be some complex valued sequences. Then $\left(a_{n}\right)_{n}$ is a Dold sequence with Dold coefficients $\left(b_{n}\right)_{n}$ if and only if

$$
\exp \left(-\sum_{n=1}^{\infty} a_{n} \frac{x^{n}}{n}\right)=\prod_{n=1}^{\infty}\left(1-x^{n}\right)^{b_{n}} .
$$

A sequence of fixed point indices of iterates turned out to be a Dold sequence.

Theorem 4.6 [5]. The sequence of fixed point indices of iterates $\left(\operatorname{ind}\left(f^{n}\right)\right)_{n}$ is a Dold sequence (provided it is well-defined). As a consequence it has a periodic expansion of the form (4.2) with integral coefficients.

The language of periodic expansion is a convenient tool that has been recently used in different contexts such as: study of the fixed point indices of an iterated map $[4,24,34]$ and minimization of the number of periodic points in homotopy class $[13,14,30]$.

In particular the sequence $\left(L\left(f^{n}\right)\right)_{n}$ is also Dold sequence (cf. [33] for different proofs of this fact). As a consequence, by Remark 4.4, we get

$$
L\left(f^{n}\right)=\sum_{k=1}^{\infty} b_{k} \operatorname{reg}_{k}(n)
$$

where $b_{k}$ are integers. 
Remark 4.7. The coefficients $b_{k}$ in the periodic expansion of Lefschetz numbers in the formula (4.4) are called Lefschetz numbers for periodic points (denoted also as $l\left(f^{k}\right)$ ) and play important role in the periodic points theory (cf. [26]). In particular, the determination of the set $L=\left\{k \in \mathbb{N}: b_{k} \neq 0\right\}$ provides valuable knowledge about the structure of periodic points obtained by confronting the information carried by $L$ with the local information about periodic points (expressed by fixed point indices at orbits). This comparison could be done either straighforwardly (Lefschetz-Hopf theorem) or by zeta function or equivalently by periodic expansion. During the past 30 years the program of determination of the set $L$ for different types of manifolds (in terms of action of induced maps on homology groups) and its application to various classes of maps has been realized. Among other cases this program was accomplished for:

- transversal maps of a compact manifold $M$ such that its rational homology is $H_{j}(M ; \mathbb{Q})=\mathbb{Q}$ if $j \in J \cup\{0\}$, and $H_{j}(M ; \mathbb{Q})=\{0\}$ otherwise, where $J$ is a subset of $\mathbb{N}$ with cardinality 1,2 or 3 [27],

- transversal maps of a compact manifold $M$ such that its rational ho$\operatorname{mology}$ is $H_{0}(M ; \mathbb{Q})=\mathbb{Q}, H_{1}(M ; \mathbb{Q})=\mathbb{Q} \oplus \mathbb{Q}$ and $H_{k}(M ; \mathbb{Q})=\{0\}$ for $k \neq 0,1[18]$,

- $C^{1}$ maps of rational exterior spaces and simple rational Hopf spaces $[10,12]$ (see also [11]), and transversal maps of some simple rational Hopf spaces [20],

- holomorphic maps of some complex compact manifolds [8].

\subsection{Periodic expansion of Lefschetz numbers of Morse-Smale diffeomor- phisms}

For the considered class of maps, i.e. Morse-Smale diffeomorphisms periodic expansion of Lefschetz numbers may be expressed by a use of roots of cyclotomic polynomials.

Definition 4.8. The $d$ th cyclotomic polynomial $\omega_{d}(z)$ is defined by the following formula:

$$
\omega_{d}(z)=\prod_{\varepsilon \in U_{d}}(z-\varepsilon),
$$

where $U_{d}$ denotes the set of all primitive $d$ th roots of unity.

Let us remark that $\omega_{d}(z)$ is an irreducible polynomial with integer coefficients of degree $\varphi(d)$, where $\varphi$ is the Euler function (i.e. $\varphi(d)$ is the number of positive integers less than or equal to $d$ that are co-prime to $d$ ). For example $\omega_{3}(z)=\frac{1-z^{3}}{1-z}=1+z+z^{2}, \operatorname{Deg}\left(\omega_{3}(z)\right)=2=\varphi(3)$.

Now, our aim is to establish the coefficients $b_{k}$ for the periodic expansion of Lefschetz numbers of iterated quasi-unipotent maps.

Let $\varepsilon_{1}, \ldots, \varepsilon_{\varphi(d)}$ be the all $d$ th primitive roots of unity. For a given $d$ we define

$$
L_{d}(n)=\varepsilon_{1}^{n}+\cdots+\varepsilon_{\varphi(d)}^{n} .
$$

The cyclotomic polynomial $\prod_{i=1}^{\varphi(d)}\left(z-\varepsilon_{i}\right)$ has integer coefficients; thus $L_{d}(n)$ is equal to $\operatorname{tr} A^{n}$, for some integer matrix $A$, having the cyclotomic 
polynomial as the characteristic polynomial. On the other hand, the sequence $\operatorname{tr} A^{n}$ for an integer matrix $A$ always satisfies Dold relations (see Theorem 3.1 .4 in [25]). As a consequence, by Theorem $1.2 L_{d}(n)$ could be uniquely represented as an integral combination of basic sequences $\operatorname{reg}_{k}$.

Let us consider a Morse-Smale diffeomorphism $f$ of a closed manifold $M$ of dimension $m$. Let $e_{i}(\lambda)$ be the algebraic multiplicity of $\lambda$ as an eigenvalue of $f_{* i}$. Define

$$
e(\lambda):=\sum_{i=0}^{m}(-1)^{i} e_{i}(\lambda) .
$$

We will call an eigenvalue $\lambda \neq 0$ essential provided $e(\lambda) \neq 0$. It is obvious that only essential eigenvalues give the contribution to $\left\{L\left(f^{n}\right)\right\}_{n=1}^{\infty}$.

Denote by $\sigma_{e s}(f)$ the set of essential eigenvalues of $f$. We define

$$
e(d)=\sum_{\lambda \in U_{d} \cap \sigma_{e s}(f)} e(\lambda) .
$$

Notice that the essential $d$ th primitive roots of unity appear in groups of $\varphi(d)$ elements, contributing $\frac{e(d)}{\varphi(d)} L_{d}(n)$ to $L\left(f^{n}\right)$. As a result we get:

$$
L\left(f^{n}\right)=\sum_{d} \frac{e(d)}{\varphi(d)} L_{d}(n) .
$$

As a consequence, to find the periodic expansion of $\left\{L\left(f^{n}\right)\right\}_{n=1}^{\infty}$ it is enough to determine the expansions of each $\left\{L_{d}(n)\right\}_{n=1}^{\infty}$.

We represent $\left\{L_{d}(n)\right\}_{n=1}^{\infty}$ as an integral combination of basic sequences $\operatorname{reg}_{k}$ :

$$
L_{d}(n)=\sum_{k=1}^{\infty} b_{k}^{d} \operatorname{reg}_{k}(n),
$$

where $b_{k}^{d}$ are integers, $d$ is fixed.

The following theorem gives the value of $b_{k}^{d}$, and thus allows us to determine the periodic expansion of $\left\{L_{d}(n)\right\}_{n=1}^{\infty}$. It was proved in [15] in an elementary but rather long way. Below we will give much simpler proof that is based on Theorem 4.5.

Theorem 4.9. The coefficient $b_{k}^{d}$ of the periodic expansion of $\left\{L_{d}(n)\right\}_{n=1}^{\infty}$ is equal to:

$$
b_{k}^{d}= \begin{cases}\mu\left(\frac{d}{k}\right) & \text { if } k \mid d, \\ 0 & \text { if } k \nmid d .\end{cases}
$$

Proof. If $a_{n}=\sum_{i=1}^{r} m_{i} \lambda_{i}^{n}$, where $m_{i}$ are integers and $\lambda_{i}$ are some complex numbers, then (cf. [25] (3.1.26)):

$$
Z_{a_{n}}(z)=\prod_{i=1}^{r}\left(1-\lambda_{i} z\right)^{-m_{i}} .
$$

On the other hand, in our case each $\lambda_{i} \in U(d)$, where $U(d)$ denotes the set of all primitive roots of unity of degree $d$ and $r=\varphi(d)$. As $\varphi(d)$ is always 
even for $d>1, U(d)$ is closed for taking the inverses and in our case each $m_{i}=1$, we get:

$Z_{L_{d}(n)}(z)=\prod_{i=1}^{\varphi(d)}\left(\lambda_{i} z-1\right)^{-1}=\prod_{i=1}^{\varphi(d)}\left(z-\frac{1}{\lambda_{i}}\right)^{-1}=\prod_{i=1}^{\varphi(d)}\left(z-\lambda_{i}\right)^{-1}=\omega_{d}(z)^{-1}$.

On the other hand, the following well-known fact holds (cf. for example $[7])$ :

$$
\omega_{d}(z)^{-1}=\prod_{k \mid d}\left(1-z^{k}\right)^{-\mu\left(\frac{d}{k}\right)} .
$$

Applying the formula (4.3) for $a_{n}=L_{d}(n)$ we get that

$$
Z_{L_{d}(n)}(z)=\prod_{k=1}^{\infty}\left(1-z^{k}\right)^{-b_{k}^{d}}
$$

Comparing the formulas (4.10) and (4.11) we get the equality (4.9).

\section{Periodic expansion of indices of iterates at periodic points for Morse-Smale diffeomorphisms}

Let us denote for short the derivative of $f$ at $x_{0} \in \operatorname{Fix}(f)$ by $D=D f\left(x_{0}\right)$ and by $\sigma(D)$ its spectrum. By $\sigma_{+}$we denote the number of real eigenvalues of $D$ greater than 1 and by $\sigma_{-}$the number of real eigenvalues of $D$ less than -1 , in both cases counting with multiplicity. We consider hyperbolic maps (i.e. maps having only hyperbolic periodic points). For a fixed point $x_{0}$ we get [4]:

$$
\operatorname{ind}\left(f^{n}, x_{0}\right)=\operatorname{sgn} \operatorname{det}\left(I d-D^{n}\right)= \begin{cases}(-1)^{\sigma_{+}} & \text {if } n \text { is odd } \\ (-1)^{\sigma_{+}+\sigma_{-}} & \text {if } n \text { is even. }\end{cases}
$$

In dependence of parity of the values $\sigma_{+}, \sigma_{-}$and $n$, we obtain four possibilities:

$$
\operatorname{ind}\left(f^{n}, x_{0}\right)=\left\{\begin{array}{l}
\operatorname{reg}_{1}(n), \\
-\operatorname{reg}_{1}(n), \\
\operatorname{reg}_{1}(n)-\operatorname{reg}_{2}(n), \\
-\operatorname{reg}_{1}(n)+\operatorname{reg}_{2}(n) .
\end{array}\right.
$$

Let us consider a point $x_{0}$ with minimal period $k$ and its orbit $O_{x_{0}}=$ $\left\{x_{0}, f\left(x_{0}\right), \ldots, f^{k-1}\left(x_{0}\right)\right\}$. Then by $(5.2)$ we obtain that there are only four possible forms of indices for the orbit:

$$
\operatorname{ind}\left(f^{n}, O_{x_{0}}\right)=\left\{\begin{array}{l}
\operatorname{reg}_{k}(n), \\
-\operatorname{reg}_{k}(n), \\
\operatorname{reg}_{k}(n)-\operatorname{reg}_{2 k}(n), \\
-\operatorname{reg}_{k}(n)+\operatorname{reg}_{2 k}(n) .
\end{array}\right.
$$

There is a one-to-one correspondence between the sequences in (5.3) and the forms of the Lefschetz zeta functions, namely the following relations hold: 


\section{Theorem 5.1.}

$$
\begin{aligned}
& \text { (A) for } a_{n}=l \operatorname{reg}_{k}(n), \quad Z_{a_{n}}(t)=\frac{1}{\left(1-t^{k}\right)^{l}} . \\
& (B) \text { for } a_{n}=l\left(\operatorname{reg}_{k}(n)-\operatorname{reg}_{2 k}(n)\right), Z_{a_{n}}(t)=\left(1+t^{k}\right)^{l} .
\end{aligned}
$$

Proof. We will consider each case separately:

(A) Let $a_{n}=l \operatorname{reg}_{k}(n)$; by Theorem 4.5 we have the following relation between sequences $a_{n}$ and $b_{n}$ :

$$
Z_{a_{n}}(t)=\exp \left(\sum_{n=1}^{\infty} \frac{a_{n}}{n} t^{n}\right)=\exp \left(\sum_{n=1}^{\infty} \frac{l \operatorname{reg}_{k}(n)}{n} t^{n}\right)=\prod_{n=1}^{\infty}\left(1-t^{n}\right)^{-l \cdot b_{n}},
$$

where $b_{n}=\frac{1}{n} \sum_{s \mid n} \mu(s) a_{\frac{n}{s}}$ and we get by the definition of $\left(\operatorname{reg}_{k}\right)_{n}$ that $b_{n}$ is equal to 1 for $n=k$, and zero otherwise, which gives us the desired form of $Z_{a_{n}}(t)$.

(B) Let $a_{n}=l\left(\operatorname{reg}_{k}(n)-\operatorname{reg}_{2 k}(n)\right)$; using the result from the previous case we have

$$
\begin{aligned}
Z_{a_{n}}(t) & =\exp \left(l \sum_{n=1}^{\infty} \frac{\operatorname{reg}_{k}(n)}{n} t^{n}\right) \exp \left(-l \sum_{n=1}^{\infty} \frac{\operatorname{reg}_{2 k}(n)}{n} t^{n}\right)=\frac{\left(1-t^{2 k}\right)^{l}}{\left(1-t^{k}\right)^{l}} \\
& =\left(1+t^{k}\right)^{l}
\end{aligned}
$$

\section{The minimal set of Lefschetz periods expressed by periodic expansions}

By Lefschetz-Hopf formula we may represent the sequence of Lefschetz numbers in the following form:

$$
L\left(f^{n}\right)=\sum_{x \in \mathrm{P}(f)} \operatorname{ind}\left(f^{n}, x\right)=\sum_{k} \sum_{O \in \operatorname{Orb}_{k}(f)} \operatorname{ind}\left(f^{n}, O\right),
$$

where $\operatorname{Orb}_{k}(f)$ denotes the set of $k$-orbits of $f$ and each $O$ has the form (5.3).

For $j \in\{1,2,3,4\}$ let us denote by $c_{r_{i}}^{j}$ an integer sequence that has one of the forms of fixed point indices of an $r_{i}$-orbit in (5.3), i.e.

$$
c_{r_{i}}^{j}(n)= \begin{cases}\operatorname{reg}_{r_{i}}(n), & j=1, \\ -\operatorname{reg}_{r_{i}}(n), & j=2, \\ \operatorname{reg}_{r_{i}}(n)-\operatorname{reg}_{2 r_{i}}(n), & j=3, \\ -\operatorname{reg}_{r_{i}}(n)+\operatorname{reg}_{2 r_{i}}(n), & j=4 .\end{cases}
$$

Proposition 6.1. The following formula holds:

$$
\operatorname{MPer}_{L}(f)=\bigcap\left\{r_{1}, r_{2}, \ldots, r_{N(f)}\right\},
$$

where the intersection is taken over all possible decompositions of $\left(L\left(f^{n}\right)\right)_{n}$ given by the formula (6.1) into the sum of sequences of the form $(6.2)$. 
Proof. Each representation of $Z_{f}(t)$ in the form (3.6) (with the factors $(1+$ $\left.\Delta_{i} t^{r_{i}}\right)^{m_{i}}$ ) is equivalent by Theorem 5.1 to the unique representation of $\left(L\left(f^{n}\right)\right)_{n}$ as the sum of the sequences of the form (6.2) (with $k=r_{i}$ representing the minimal period of an orbit).

Theorem 6.2. The following formula holds:

$\left.\operatorname{MPer}_{L}(f)=\left\{k: b_{k} \neq 0 \text { in the periodic expansion (4.4) of } L\left(f^{n}\right)\right)_{n}\right\} \cap O d d$,

where Odd denotes the set of odd natural numbers.

Proof. It is obvious that for odd $k$ for which $b_{k} \neq 0$ in (4.4) we get that $k \in \operatorname{MPer}(f)$. We show that there are no even numbers in $\operatorname{MPer}(f)$. Assume, contrary to our claim, that there is an even $r_{i}=2 u$ in $\operatorname{MPer}_{L}(f)$. Then for every decomposition of $L\left(f^{n}\right)$ into (6.2) there must be the term $c_{2 u}^{j}(n)$ for some $j$. However, for $j=1$ we have

$$
c_{2 u}^{1}(n)=\operatorname{reg}_{2 u}(n)=\left(\operatorname{reg}_{2 u}(n)-\operatorname{reg}_{u}(n)\right)+\operatorname{reg}_{u}(n)=c_{u}^{4}(n)+c_{u}^{2}(n) .
$$

For $j=3$ there is:

$$
\begin{aligned}
c_{2 u}^{3}(n) & =\operatorname{reg}_{2 u}(n)-\operatorname{reg}_{4 u}(n)=\left(\operatorname{reg}_{2 u}(n)-\operatorname{reg}_{u}(n)\right)+\operatorname{reg}_{u}(n)-\operatorname{reg}_{4 u}(n)= \\
& =c_{u}^{3}(n)+c_{u}^{1}(n)+c_{4 u}^{1}(n) .
\end{aligned}
$$

Analogously, for $j=2,4$ we obtain another decompositions of $c_{2 u}^{j}(n)$, which contradicts our assumption.

Remark 6.3. Theorem 6.2 holds in fact for a larger class of maps, namely for maps having finitely many periodic points, all of them hyperbolic. For a map $f$ in this class the sequence $\left(L\left(f^{n}\right)\right)_{n}$ is bounded (cf. [4]) and thus the only non-zero eigenvalues of $f_{*}$ which give the contribution to $\left(L\left(f^{n}\right)\right)_{n}$ (i.e. have different multiplicity in odd and even homology) are roots of unity [1]. As a consequence, the analysis of such maps reduces to quasi-unipotent case.

\section{Applications: the minimal sets of Lefschetz periods on $N_{g}$, a non-orientable compact surface without boundary of genus $g$}

Let us remind that $N_{g}$ is homeomorphic with the connected sum of $g$ real projective planes and its homology groups are the following: $H_{0}(M, \mathbb{Q})=\mathbb{Q}$, $H_{2}(M, \mathbb{Q})=0$ and

$$
H_{1}(M, \mathbb{Q})=\underbrace{\mathbb{Q} \oplus \cdots \oplus \mathbb{Q}}_{g-1} .
$$

\subsection{Realization of the minimal set of Lefschetz periods on $N_{g}$}

Conjecture 7.1. Llibre and Sirvent [28] formulated the following conjecture: can any finite set of odd positive integers be the minimal set of Lefschetz periods for a $C^{1}$ Morse-Smale diffeomorphism on some non-orientable compact surface without boundary with a convenient genus? 
We will show that there are no algebraic obstacles on the homology which would prevent the validity of Conjecture 7.1.

Let $d$ be a degree of some primitive root of unity; we take maximal $m$ such that $2 m-1 \leq d$ and denote by $c^{d}$ the vector $\left[b_{1}^{d}, b_{3}^{d}, \ldots, b_{2 m-1}^{d}, 0, \ldots\right]$, where $b_{k}^{d}$ is given by the formula (4.9), i.e. $b_{k}^{d}=\left\{\begin{array}{ll}\mu\left(\frac{d}{k}\right) & \text { if } k \mid d, \\ 0 & \text { if } k \nmid d .\end{array}\right.$.

Observe that for odd $k$ there is $b_{k}^{d}=-b_{k}^{2 d}$ and as a consequence,

$$
c^{d}=-c^{2 d} \text {. }
$$

Theorem 7.2. The set $\mathbb{A}_{m}=\left\{c^{1}, c^{3}, c^{5}, \ldots, c^{(2 m-1)}\right\}$ of $m$ vectors is a basis of $\mathbb{Z}^{m}$.

Proof. We identify $c^{d}$ with $\left[b_{1}^{d}, b_{3}^{d} \ldots, b_{2 m-1}^{d}\right]$. Consider the matrix $A$ composed of vectors $c^{i}$ in rows:

$$
\begin{aligned}
& c^{1} \leftrightarrow \operatorname{reg}_{1} \\
& c^{3} \leftrightarrow-\operatorname{reg}_{1}+\operatorname{reg}_{3} \\
& c^{5} \leftrightarrow-\operatorname{reg}_{1}+\operatorname{reg}_{5} \\
& \vdots \\
& c^{2 m-1} \leftrightarrow \ldots
\end{aligned}\left[\begin{array}{ccccc}
b_{1} & b_{3} & b_{5} & \ldots & b_{2 m-1} \\
-1 & 0 & 0 & \ldots & 0 \\
-1 & 0 & 0 & \ldots & 0 \\
\vdots & \vdots & \vdots & \ddots & 0 \\
. & . & . & . & \vdots \\
\end{array}\right]=A
$$

Note that $b_{d}^{d}=\mu\left(\frac{d}{d}\right)=1$ and for $i>d$ we have $b_{i}^{d}=0$. As a consequence, the matrix $A$ is lower triangular and $\operatorname{det} A=1$, so the set $\mathbb{A}_{m}$ is a basis of $\mathbb{Z}^{m}$.

Theorem 7.3. For any finite set $\mathcal{G}_{m}=\left\{a_{1}, a_{3}, \ldots, a_{2 m-1}\right\}$ of $m$ integers there exists quasi-unipotent map $f_{*}: H_{*}\left(N_{g}\right) \rightarrow H_{*}\left(N_{g}\right)$ such that

(a) $L\left(f_{*}^{n}\right)=\operatorname{tr}\left(f_{*_{0}}^{n}\right)-\operatorname{tr}\left(f_{*_{1}}^{n}\right)=-\sum_{i=1}^{2(2 m-1)} a_{i} \operatorname{reg}_{i}(n)$,

(b) $\operatorname{MPer}_{L}\left(f_{*}\right)=\left\{i: a_{i} \neq 0 \wedge a_{i} \in \mathcal{G}_{m}\right\}$,

Proof. (a) Remind that by the formula (4.9) Lefschetz numbers of self-map of $N_{g}$ have the form

$$
L\left(f^{n}\right)=\operatorname{tr}\left(f_{*_{0}}^{n}\right)-\operatorname{tr}\left(f_{*_{1}}^{n}\right)=\operatorname{reg}_{1}-\sum_{i} \sum_{k=1}^{\infty} b_{k}^{d_{i}} \operatorname{reg}_{k}(n) .
$$

We are searching for a homomorphism $f_{*}$ satisfying for each odd $i$ the equality:

$$
L\left(f_{*}^{n}\right)=-\sum_{i=1}^{2 m-1} a_{i} \operatorname{reg}_{i}(n)=\underbrace{\operatorname{reg}_{1}(n)}_{\operatorname{tr}\left(f_{*_{0}}^{n}\right)}-\underbrace{\left(\left(a_{1}+1\right) \operatorname{reg}_{1}(n)+\sum_{i=2}^{2 m-1} a_{i} \operatorname{reg}_{i}(n)\right)}_{\operatorname{tr}\left(f_{* 1}^{n}\right)} .
$$

Consider the vector $\left[a_{1}+1, a_{3}, \ldots, a_{2 m-1}\right]$ of odd indices of coefficients coming from $\operatorname{tr}\left(f_{*_{1}}\right)$. By Theorem 7.2 there exists unique representation of this vector in the basis $\left\{c^{1}, c^{3}, \ldots c^{2 m-1}\right\}$ such that:

$$
\left[a_{1}+1, a_{3}, \ldots, a_{2 m-1}\right]=\sum_{i=1}^{m} m_{i} c^{2 i-1}=\sum_{i=1}^{m}\left|m_{i}\right| c^{s(i)}
$$




$$
\text { for } s(i)=\left\{\begin{array}{l}
2 i-1 \quad \text { if } m_{i}>0, \\
2(2 i-1) \text { if } m_{i}<0,
\end{array}\right. \text { where in the last equality we used }
$$
the relation (7.1). Now we define the map $f_{*_{1}}$ represented by a diagonal matrix of dimension

$$
D=\sum_{i=1}^{m}\left|m_{i}\right| \varphi(2 i-1),
$$

which have on the diagonal: $\left|m_{i}\right|$ copy of all primitive $(2 i-1)$-roots of unity for each $m_{i} \geqslant 0$, and $\left|m_{i}\right|$ copies $2(2 i-1)$-roots of unity for each $m_{i}<0$, where $i=1, \ldots, m$.

(b) follows directly from $(a)$ and Theorem 6.2 .

Remark 7.4. Let us notice that Conjecture 7.1 coincides with the item (b) of Theorem 7.3, while item (a) is more general. What is more, it provides the bound for the dimension of the realization (7.2) as well as the bound for the highest degree of roots of unity needed in the realization $(2(2 m-1))$.

Corollary 7.5. Lefschetz zeta function of the realization $f_{*}$ has the following form:

$$
Z_{f_{*}}(t)=\frac{1}{t-1} \prod_{i}\left(\prod_{q \mid s(i)}\left(1-z^{q}\right)^{-\mu\left(\frac{s(i)}{q}\right)}\right)^{\left|m_{i}\right|} .
$$
Thus

Indeed, observe that $Z_{f_{*}}(t)=Z_{l_{n}}(t)$, where $l_{n}=1-\sum_{i}\left|m_{i}\right| L_{s(i)}(n)$.

$$
Z_{f_{*}}(t)=\frac{1}{t-1} \prod_{i}\left(Z_{L_{s(i)}}(t)\right)^{\left|m_{i}\right|},
$$

and by Theorem 4.11 we get the formula (7.3).

Remark 7.6. There is a "topological" part of Conjecture 7.1 which is still unsolved. Namely, it remains an open question whether the quasi-unipotent map $f_{*}$ found in Theorem 7.3 could be realized as a map that is induced by some Morse-Smale diffeomorphism on $N_{g}$.

\subsection{Algorithmic approach to determine the minimal sets of Lefschetz periods for self-maps of $N_{g}$}

In this section we describe a simple algorithm that enables us to determine the minimal sets of Lefschetz periods for self-maps of $N_{g}$. As an application we use a computer program based on this algorithm to verify $\operatorname{MPer}_{L}(f)$ for $g<10$ found in [28] and to compute $\operatorname{MPer}_{L}(f)$ for $g \geq 10$.

We will sketch below the main scheme of the algorithm, while the detailed description is placed in Appendix. The program for computation of $\operatorname{MPer}_{L}(f)$ based on the algorithm (in Mathematica) is available on the webpage of Myszkowski. ${ }^{1}$

We assume that $g>1$; otherwise the matrix $f_{*_{1}}$ has the dimension 0 and the only possibility is $\operatorname{MPer}_{L}(f)=\{1\}$. The dimension of the matrix $f_{*_{1}}$

\footnotetext{
${ }^{1}$ http://www.mif.pg.gda.pl/homepages/amyszkowski/.
} 
is $g-1$ and all eigenvalues are primitive $d_{i}$-roots of unity grouped in $\varphi\left(d_{i}\right)$ elements. Consequently $g-1=\sum_{i} \varphi\left(d_{i}\right)$.

We define a finite family $P$ of partitions $P_{j}$ of the number $g-1$ into possible values of $\varphi$, i.e.

$$
P=\left\{P_{j}=\bigcup_{i}\left\{d_{i}\right\}: g-1=\sum_{i} \varphi\left(d_{i}\right), d_{i} \in \mathbb{N}\right\} .
$$

The next lemma allows us to find the bound for the number of elements in the family $P$.

Lemma 7.7 (cf. [31]). Let $\varphi$ be the Euler function. For all $n \in \mathbb{N}$, with the exception of $n=2,4,6,10,12,18,30$ we have

$$
\varphi(n) \geqslant n^{\log _{3} 2} \text {. }
$$

Corollary 7.8. Let $\varphi$ be the Euler function. For all $n \in \mathbb{N}$ we have

$$
\varphi(n) \geqslant\left(\frac{n}{2}\right)^{\log _{3} 2} .
$$

By application of the inequality (7.4) we get that:

$$
P=\left\{\bigcup_{i}\left\{d_{i}\right\}: g-1=\sum_{i} \varphi\left(d_{i}\right), d_{i} \leqslant 2(g-1)^{\log _{2} 3}, d_{i} \in \mathbb{N}\right\} .
$$

We can associate with each partition $P_{j}$ (i.e. each set of degrees) the corresponding sequence of Lefschetz numbers in the form of a periodic expansion:

$$
L\left(f^{n}\right)=1-\sum_{d_{i} \in P_{j}} L_{d_{i}}(n)=\operatorname{reg}_{1}(n)-\sum_{d_{i} \in P_{j}} \sum_{k} b_{k}^{d_{i}} \operatorname{reg}_{k}(n),
$$

where $b_{k}^{d_{i}}$ are defined by the formula (4.9) of Theorem 4.9. By Theorem 6.2 the set of minimal periods $M_{P_{j}}$ for a map corresponding to a group of degrees $P_{j}$ is

$$
\begin{aligned}
M_{P_{j}}= & \left\{k: \sum_{i=1}^{\left|P_{j}\right|} a_{k}^{d_{i}} \neq 0,2 \nmid k, d_{i} \in P_{j}\right\}, \quad \text { where } a_{1}^{d_{i}}=b_{1}^{d_{i}}-1, \\
& a_{k}^{d_{i}}=b_{k}^{d_{i}} \text { for } k>1 .
\end{aligned}
$$

Finally, the family of the minimal sets of Lefschetz periods $\operatorname{MPer}_{L}(f)$ over all $f$ being Morse-Smale diffeomorphisms of $N_{g}$ is given as follows:

$$
\operatorname{MPer}_{L}=\bigcup_{j=1}^{|P|}\left\{M_{P_{j}}\right\}
$$


TABLE 1. The family of all minimal sets of Lefschetz periods for Morse-Smale diffeomorphism of $N_{4}$

\begin{tabular}{lll}
\hline$P_{j}$ & $L\left(f^{n}\right)$ & $M_{P_{j}}=\operatorname{MPer}_{L}(f)$ \\
\hline$\{1,3\}$ & $\operatorname{reg}_{1}(n)-\operatorname{reg}_{3}(n)$ & $\{1,3\}$ \\
$\{1,4\}$ & $\operatorname{reg}_{2}(n)-\operatorname{reg}_{4}(n)$ & $\emptyset$ \\
$\{1,6\}$ & $-\operatorname{reg}_{1}(n)+\operatorname{reg}_{2}(n)+\operatorname{reg}_{3}(n)-\operatorname{reg}_{6}(n)$ & $\{1,3\}$ \\
$\{2,3\}$ & $3 \operatorname{reg}_{1}(n)-\operatorname{reg}_{2}(n)-\operatorname{reg}_{3}(n)$ & $\{1\}$ \\
$\{2,4\}$ & $2 \operatorname{reg}_{1}(n)-\operatorname{reg}_{4}(n)$ & $\{1\}$ \\
$\{2,6\}$ & $\operatorname{reg}_{1}(n)+\operatorname{reg}_{3}(n)-\operatorname{reg}_{6}(n)$ & $\{1,3\}$ \\
$\{1,1,1\}$ & $-2 \operatorname{reg}_{1}(n)$ & $\{1\}$ \\
$\{1,1,2\}$ & $-\operatorname{reg}_{2}(n)$ & $\emptyset$ \\
$\{1,2,2\}$ & $2 \operatorname{reg}_{1}(n)-2 \operatorname{reg}_{2}(n)$ & $\{1\}$ \\
$\operatorname{MPer}_{L}$ & & $\{\emptyset,\{1\},\{3\},\{1,3\}\}$ \\
\hline
\end{tabular}

TABLE 2. Selected calculations of the family $\operatorname{MPer}_{L}$ of the minimal sets of Lefschetz periods for Morse-Smale diffeomorphism of $N_{g}$ computed on CPU: Phenom II x4 965

\begin{tabular}{lll}
\hline Genus & MPer $_{L}$ & Time of execution (s) \\
\hline 9 & $\{\{1\},\{3\},\{5\},\{7\},\{9\},\{1,3\},\{1,5\}$, & 0.00572536 \\
& $\{1,7\},\{1,9\},\{3,5\},\{3,9\},\{1,3,5\}$, & \\
& $\{1,3,7\},\{1,3,9\},\{3,5,15\},\{1,3,5$, & \\
& $15\}\}$ & \\
& & \\
10 & \{\}$,\{1\},\{3\},\{5\},\{1,3\},\{1,5\},\{1,7\}$, & 0.00690033 \\
& $\{1,9\},\{3,5\},\{3,7\},\{3,9\},\{1,3,5\}$, & \\
& $\{1,3,7\},\{1,3,9\},\{1,3,5,15\}\}$ & \\
30 & Number of lists: 363 & 0.986523 \\
40 & Number of lists: 1230 & 10.7953 \\
50 & Number of lists: 3568 & 117.685 \\
\hline
\end{tabular}

Example 7.9. Let $g=4$; then $\operatorname{dim} f_{*_{1}}=3$. There are 9 possible families $P_{j}$ of degrees of primitive roots of unity (each corresponding to some induced map $\left.f_{*}\right)$. In Table 1 the set $M_{P_{j}}=\operatorname{MPer}_{L}(f)$ is described for each $P_{j}$ and $\operatorname{MPer}_{L}$ is determined.

Comparing the results of Theorem 8 in [28] for $g=9$ with the computations based on our algorithm (see Table 2) one can observe that in [28] the case $\{3,5\}$ is omitted. We will show straightforwardly the realization of this set in two ways. 
Consider $f_{*}$ that corresponds to the set $\{5,6,6\}$ of degrees of primitive roots of unity (satisfying the equation $g-1=8=\varphi(5)+\varphi(6)+\varphi(6)$ ). We have in the terms of periodic expansion

$$
\begin{aligned}
L\left(f^{n}\right)= & \operatorname{reg}_{1}(n)-\left(L_{5}(n)+L_{6}(n)+L_{6}(n)\right)=-2 \operatorname{reg}_{2}(n)-2 \operatorname{reg}_{3}(n) \\
& +\operatorname{reg}_{5}(n)-2 \operatorname{reg}_{6}(n)
\end{aligned}
$$

and thus by Theorem $6.2 \operatorname{MPer}_{L}(f)=\{3,5\}$.

Alternatively, using the language of Lefschetz zeta function, we obtain

$$
c_{5}(t)=\frac{1-t^{5}}{1-t} \quad c_{6}(t)=\frac{1+t^{3}}{1+t} .
$$

Thus. the zeta Lefschetz function for $f$ has the possible forms

$$
Z_{f}(t)=\frac{\left(1+t^{3}\right)^{2}\left(1-t^{5}\right)}{\left(1-t^{2}\right)^{2}}=\frac{\left(1+t^{3}\right)^{2}\left(1-t^{5}\right)}{(1-t)^{2}(1+t)^{2}}=\frac{\left(1+t^{3}\right)^{2}\left(1-t^{5}\right)}{(1-t)(1+t)\left(1-t^{2}\right)} .
$$

Finally, according to Definition 3.3 we get that $\operatorname{MPer}_{L}(f)=\{2,3,5\} \cap$ $\{1,3,5\} \cap\{1,2,3,5\}=\{3,5\}$.

Open Access. This article is distributed under the terms of the Creative Commons Attribution 4.0 International License (http://creativecommons.org/licenses/by/4. 0/), which permits unrestricted use, distribution, and reproduction in any medium, provided you give appropriate credit to the original author(s) and the source, provide a link to the Creative Commons license, and indicate if changes were made.

Publisher's Note Springer Nature remains neutral with regard to jurisdictional claims in published maps and institutional affiliations.

\section{Appendix: The algorithm for the determination of $\mathrm{MPer}_{L}$}

For the sake of clarity we divided our algorithm into some steps (Algorithms 1-4 below). In Algorithm 1 we find the set $\varphi\left(\varphi^{-1}[1, g-1]\right)$ by using the function PhiValues.

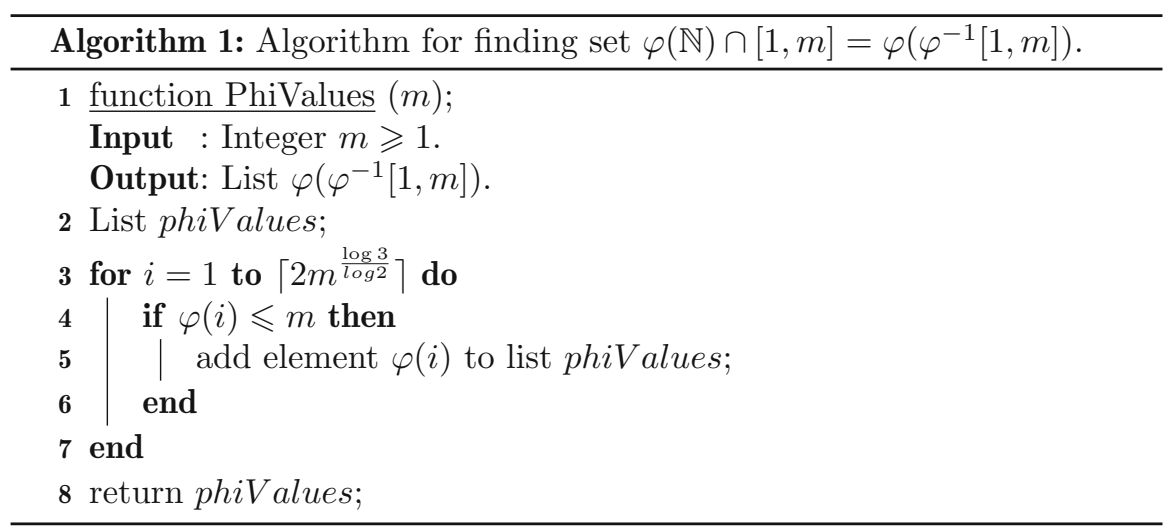

Example 8.1. Input: $m=4$; Output: PhiValues $(4)=\{1,2,4\}$.

Algorithm 2 groups degrees of primitive roots of unity by their Euler function value. 


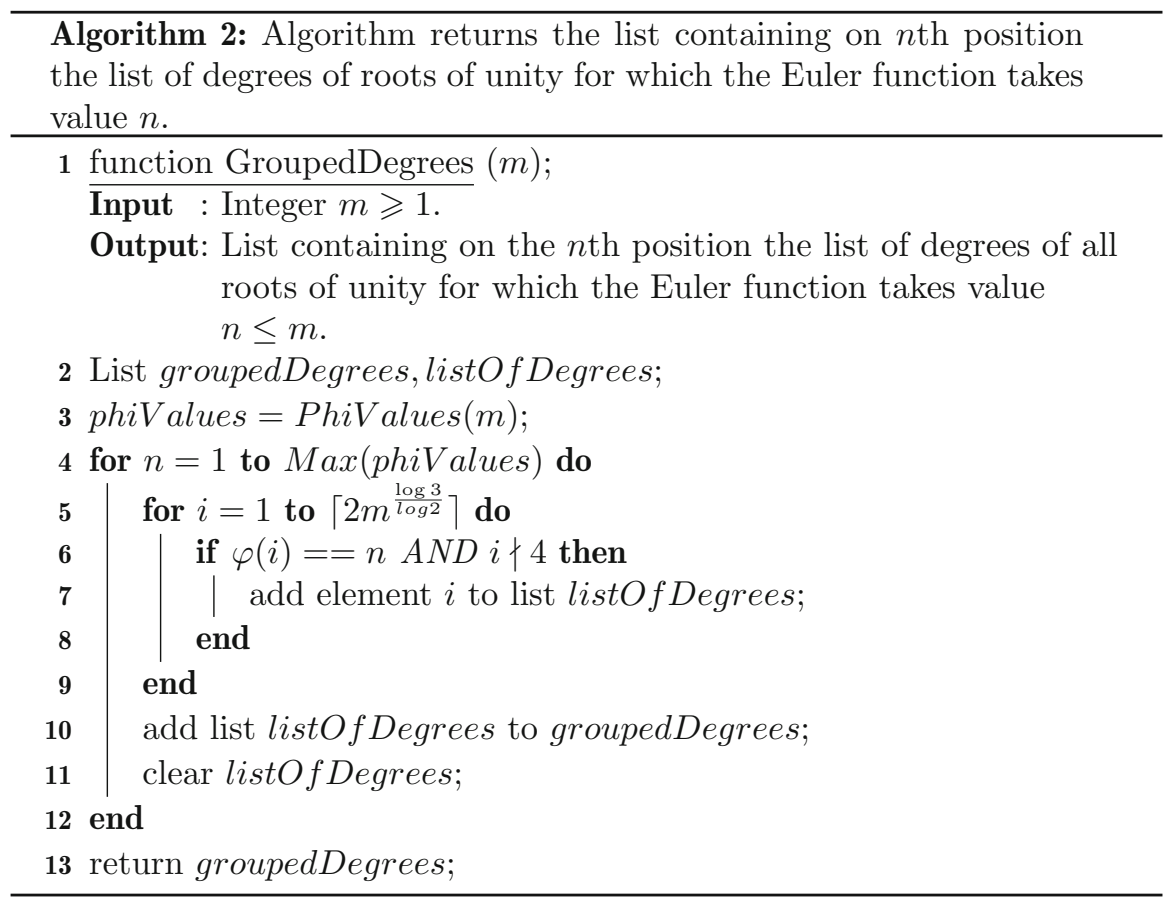

Example 8.2.

$$
\text { Input: } m=4 \text {; Output: }\{\{\underbrace{1,2}_{\begin{array}{c}
\varphi(d)=1 \\
d \nmid 4
\end{array}}\},\{\underbrace{3,6}_{\begin{array}{c}
\varphi(d)=2 \\
d \nmid 4
\end{array}}\}, \underbrace{\{\}}_{\begin{array}{c}
\varphi(d)=3 \\
d \nmid 4
\end{array}},\{\underbrace{5,10}_{\substack{\varphi(d)=4 \\
d \nmid 4}}\} .
$$

Algorithm 3 determines a list of odd coefficients of $b_{k}^{d}$ for every integer $d$ from GroupedDegrees $(m)$.

Note that $\varphi(2 k)=\varphi(2) \varphi(k)=\varphi(k)$ for odd number $k$. Hence we can write them in the list of length $\max \{\operatorname{PhiValues}(m)\} / 2$

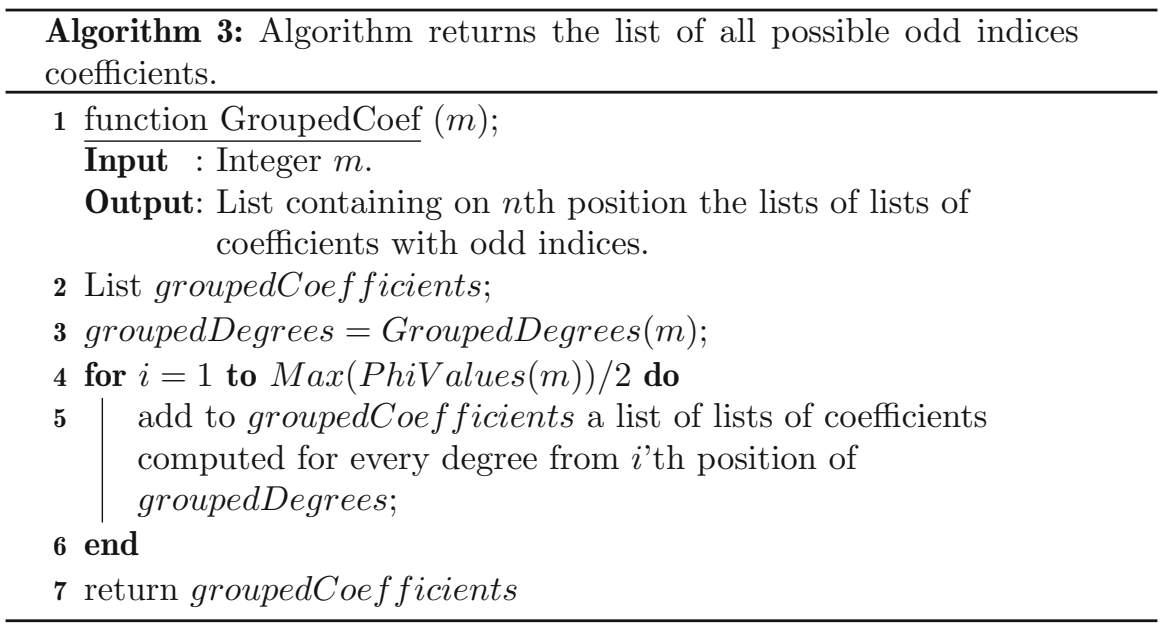


Example 8.3. Input: $m=4$; Output: $\{\{\{1,0,0\},\{-1,0,0\}\},\{\{-1,1$, $0\},\{1,-1,0\}\},\{\},\{\{-1,0,1\},\{1,0,-1\}\}\}$.

Definition 8.4. Let $A, B \subset \mathbb{R}^{n}$, the Minkowski sum of $A$ and $B$ is the set

$$
\{a+b: a \in A, b \in B\} .
$$

In the final Algorithm 4 we will use some well-known functions introduced below:

- MinkowskiSum $(A, B)$ which calculates Minkowski sum of the lists $A$, B. (for example MinkowskiSum $(\{\{1,2\},\{3,4\}\},\{\{5,6\}\})=\{\{6,8\}$, $\{8,10\}\})$,

- IntegerPartitions $(m, A)$ which gives the list of all possible partitions of an integer $m$ into the sum of integers from the list $A$,

(for example IntegerPartitions $(4,\{1,2,4\})=\{\{4\},\{2,2\},\{2,1,1\}$, $\{1,1,1,1\}\})$,

- ChangeToOdd which changes the number $n$ to $2 n-1$ (for example ChangeToOdd $(3)=5)$.

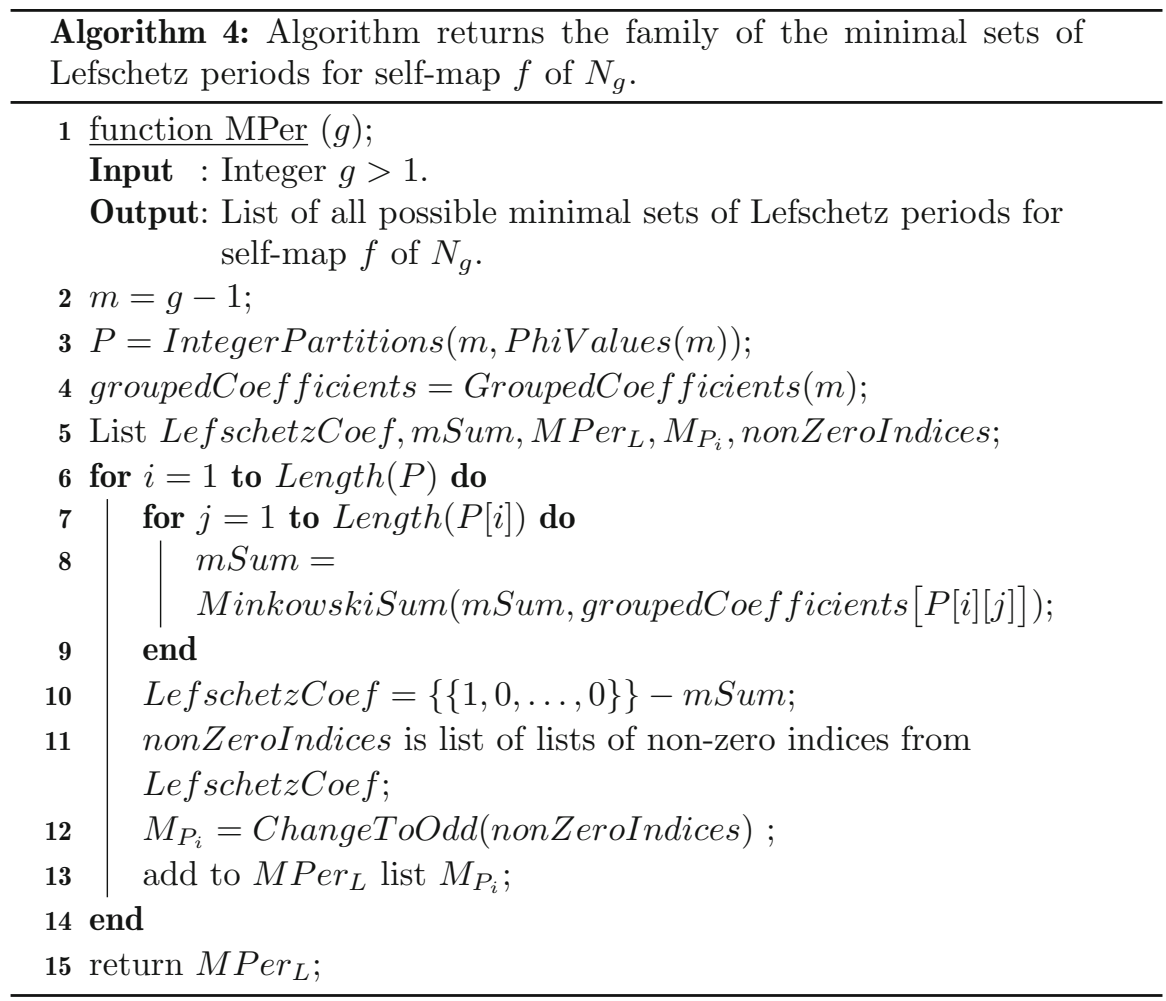

Example 8.5. Input: $g=5$; Output: $\{\{1\},\{3\},\{5\},\{1,3\},\{1,5\}\}$. 


\section{References}

[1] Babenko, I.K., Bogatyi, S.A.: The behavior of the index of periodic points under iterations of a mapping. Math. USSR Izv. 38, 1-26 (1992)

[2] Barge, H., Wójcik, K.: Mayer-Vietoris property of the fixed point index. Topol. Methods Nonlinear Anal. 50(2), 643-667 (2017)

[3] Berrizbeitia, P., González, M., Sirvent, V.: On the Lefschetz zeta function and the minimal sets of Lefschetz periods for Morse-Smale diffeomorphisms on products of $l$-spheres. Topol Appl. 235, 428-444 (2018)

[4] Chow, S. N., Mallet-Parret, J., Yorke, J. A.: A periodic point index which is a bifurcation invariant, Geometric dynamics (Rio de Janeiro, 1981), pp. 109-131, Springer Lecture Notes in Math. 1007, (Berlin, 1983)

[5] Dold, A.: Fixed point indices of iterated maps. Invent. Math. 74, 419-435 (1985)

[6] Du, B.-S., Huang, S.-S., Li, M.-C.: Newton, Fermat, and exactly realizable sequences. J. Integer Seq. 8, Article 05.1.2 (2005)

[7] Dummit, D.S., Foote, R.M.: Abstract Algebra, vol. 3. Prentice Hall, Englewood Cliffs, N.J. (1991)

[8] Fagella, N., Llibre, J.: Periodic points of holomorphic maps via Lefschetz numbers. Trans. Am. Math. Soc. 352(10), 4711-4730 (2000)

[9] Franks, J.: Some smooth maps with infinitely many hiperbolic points. Trans. Am. Math. Soc. 226, 175-179 (1977)

[10] Graff, G.: Algebraic periods of self-maps of a rational exterior space of rank 2. Fixed Point Theory Appl. 2006, 80521 (2006). https://doi.org/10.1155/FPTA/ 2006/80521

[11] Graff, G.: Existence of $\delta_{m}$-periodic points for smooth maps of compact manifold. Hokkaido Math. J. 29(1), 11-21 (2000)

[12] Graff, G.: Minimal periods of maps of rational exterior spaces. Fund. Math. 163(2), 99-115 (2000)

[13] Graff, G.: Minimal number of periodic points for smooth self-maps of twoholed 3-dimensional closed ball. Topol. Methods Nonlinear Anal. 33(1), 121$130(2009)$

[14] Graff, G., Jezierski, J.: Minimal number of periodic points of smooth boundarypreserving self-maps of simply-connected manifolds. Geom. Dedicata 187, 241$258(2017)$

[15] Graff, G., Kaczkowska, A.: Reducing the number of periodic points in the smooth homotopy class of a self-map of a simply-connected manifold with periodic sequence of Lefschetz numbers. Ann. Polon. Math. 107(1), 29-48 (2013)

[16] Graff, G., Lebiedź, M., Nowak-Przygodzki, P.: Generating sequences of Lefschetz numbers of iterates. Monatsh. Math. 188(3), 511-525 (2019)

[17] Grines, V. Z., Medvedev, T. V., Pochinka, O. V.: Dynamical systems on 2- and 3-manifolds. Dev. Math. Springer (2016)

[18] Guillamon, A., Jarque, X., Llibre, J., Ortega, J., Torregrosa, J.: Periods for transversal maps via Lefschetz numbers for periodic points. Trans. Am. Math. Soc. 347(12), 4779-4806 (1995)

[19] Guirao, J.L., Llibre, J.: On the set of periods for the Morse-Smale diffeomorphisms on the disc with $n$ holes. J. Diff. Equ. Appl. 19(7), 1161-1173 (2013)

[20] Guirao, J.L., Llibre, J.: Periodic structure of transversal maps on $C P^{n}, H P^{n}$ and $S^{p} \times S^{q}$. Qual. Theory Dyn. Syst. 12(2), 417-425 (2013) 
[21] Guirao, J.L., Llibre, J.: Periods of Morse-Smale diffeomorphisms of $S^{n}$. Colloq. Math. 110(2), 477-483 (2008)

[22] Guirao, J. L., Llibre, J.: The set of periods for the Morse-Smale diffeomorphisms on $T^{2}$, Dyn. Contin. Discrete Impuls. Syst. Ser. A Math. Anal. 19(4), 471-484 (2012)

[23] Guirao, J.L., Llibre, J.: Minimal Lefschetz sets of periods for Morse-Smale diffeomorphisms on the $n$-dimensional torus. J. Diff. Equ. Appl. 16(5-6), 689703 (2010)

[24] Hernández-Corbato, L., Ruiz del Portal, F.: Fixed point indices of planar continuous maps. Discrete Contin. Dyn. Syst. 35(7), 2979-2995 (2015)

[25] Jezierski, J., Marzantowicz, W.: Homotopy methods in topological fixed and periodic points theory. Topological fixed point theory and its applications, Vol. 3, Springer, Dordrecht (2006)

[26] Llibre, J.: Lefschetz numbers for periodic points, Contemporary Math. 152, Amer. Math. Soc., Providence, RI, pp. 215-227 (1993)

[27] Llibre, J., Paranõs, J., Rodriguez, J.A.: Periods for transversal maps on compact manifolds with a given homology. Houston J. Math. 24, 397-407 (1998)

[28] Llibre, J., Sirvent, V.F.: Minimal sets of periods for Morse-Smale diffeomorphisms on non-orientable compact surfaces without boundary. J. Diff. Equ. Appl. 19(3), 402-417 (2013)

[29] Llibre, J., Sirvent, V.F.: Minimal sets of periods for Morse-Smale diffeomorphisms on orientable compact surfaces. Houston J. Math. 35(3), 835-855 (2009)

[30] Marzantowicz, W., Nowak-Przygodzki, P.: Finding periodic points of a map by use of a $k$-adic expansion. Discrete Contin. Dyn. Syst. 5(3), 495-514 (1999)

[31] Shapiro, H.: An arithmetic function arising from the $\varphi$ function. Am. Math. Monthly 50, 18-30 (1943)

[32] Shub, M.: Morse-Smale Diffeomorphisms are Unipotent on Homology, Dynamical systems (Proc. Sympos., Univ. Bahia, Salvador, 1971). Academic Press, New York (1973)

[33] Steinlein, H.: Fermat's little theorem and Gauss congruence: matrix versions and cyclic permutations. Am. Math. Monthly 124(6), 548-553 (2017)

[34] Wójcik, K.: Binomial transform and Dold sequences. J. Integer Seq. 18(1), Article 15.1.1 (2015)

Grzegorz Graff and Adrian Myszkowski

Faculty of Applied Physics and Mathematics

Gdańsk University of Technology

Narutowicza 11/12

80-233 Gdańsk

Poland

e-mail: grzegorz.graff@pg.edu.pl 


\author{
Adrian Myszkowski \\ e-mail: adrian.myszkowski@pg.edu.pl \\ Małgorzata Lebiedź \\ Institute of Mathematics \\ University of Gdańsk \\ Wita Stwosza 57 \\ 80-952 Gdańsk \\ Poland \\ e-mail: malgosilla@gmail.com
}

